\title{
Spotlight needed on Italian policy
}

Sir — The battle about the long-awaited reform of Italian research policy ${ }^{1}$ is taking place behind closed doors, and the scientific community is in the dark. There is no indication that there will be a transparent system of peer review ${ }^{2}$ in the near future. Indeed, any discussion of reform will continue to be abstract and ineffective so long as there are no objective indicators of scientific performance.

In the past 15 years, panels and committees have been set up to evaluate the scientific performance of governmentfunded research organizations, but neither detailed reports nor action have followed. Even the recent working group of the Ministry of Universities and Research ${ }^{3}$ has provided no more than a laudable declaration of intent and a comprehensive catalogue of scientific centres, research activities and numbers of employees.

The 71 pages of graphs and comments provide no data on cash flow compared to scientific production per research centre and scientific project, or basic information on how, where and with what results money is spent.

This is unfortunate; a fast, reasonably objective and cost-effective way of measuring the scientific performance of research institutions is to use bibliometric indicators ${ }^{4}$. Statistics such as the number of publications and their relative impact factors can show quite surprising results. For instance, in a pilot study ${ }^{5}$ we have scrutinized the publication output of four main categories of scientific institutions in the field of environmental research: universities, CNR (National Research Council), ENEA (National Agency for New Technologies, Energy and the Environment), and all the other smaller organizations.

From the results reported in Table 1, Alison Abbott's claim that ENEA should "have less to fear" from scientific reform is disputable ${ }^{1}$. In fact, we are still waiting to see the "thousand flowers" bloom as was claimed by ENEA's president, Nicola Cabibbo - the quality and productivity of ENEA research in the environmental field are among the lowest to be found in Italian science.

The Ministry of Universities and Research could easily extend this study to other scientific fields and compare the research performances of different scientific institutions and research fields both within Italy and perhaps with those of other European countries. We are aware that bibliometric indicators are not exempt from criticism ${ }^{6,7}$ and that they should be used with care in conjunction with
Table 1 Performance of Italian scientific institutions in the environmental research field

Institutions Article share AverageNo. Average of citations impact per article factor

$\begin{array}{llll}\text { Universities } & 55.3 \% & 3.94 & 1.25\end{array}$

$\begin{array}{llll}\text { CNR } & 15.9 \% & 4.68 & \ldots \ldots \ldots \ldots \ldots \ldots \ldots \ldots \ldots\end{array}$

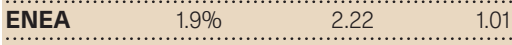

$\begin{array}{llllllllll} & \text { Other } & 26.9 \% & 3.89 & 1.34\end{array}$

These statistics ${ }^{3}$ have been computed from 4,858 articles published between 1981 and 1995 in 231 leading journals selected from environment-related categories of Scisearch ${ }^{8}$.

strategic, cultural and political

considerations. But it cannot be denied that they can bring objective and valuable information to bear on the waste of resources within the system.

Yet, after years of discussions, the bogeyman of peer review based on bibliometric indicators still comes up against ingrained fears and strenuous resistance, because of its potential to reveal structural inefficiencies and, consequently, to trigger disturbing conflicts and bring the discussion under the spotlight of public attention.

Giulio De Leo

Gianmarco Paris

Marino Gatto

Dipartimento di Elettronica

e Informazione,

Politecnico di Milano,

Piazza Leonardo da Vinci 32,

20133 Milano, Italy

e-mail:paris@dsa.unipr.it

Paolo Menozzi

Dipartimento di Scienze Ambientali,

Università degli Studi,

Viale delle Scienze,

43100 Parma, Italy

Sir - Biologists in the university sector share Ben Miflin's pride and pleasure that fellow biologists in $15 \mathrm{UK}$ research institutes have average citation rates for their papers that exceed those of their peers in the United States, Canada, Germany and France ${ }^{4}$.

That, however, is the correct comparison, institute with institute, like with like. Miflin's attempt to show that biologists in UK institutes are somehow more successful than biologists in UK universities is misguided (and invidious). He does not compare like with like.

The two types of organization and their staffs have different roles in our society and contribute differently to the progress of biological science.

\section{Peter Ayres}

(Chair, Heads of University

Biological Sciences)

Department of Biological Sciences,

Institute of Environmental

and Nature Sciences,

Lancaster University,

Lancaster LA1 4YQ, UK

e-mail:p.ayres@lancaster.ac.uk

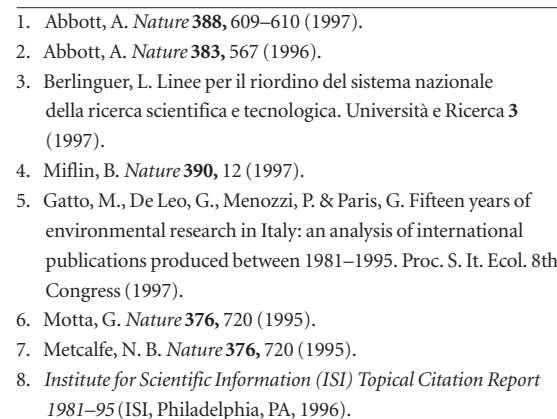

\section{Life, love and soil}

Sir - Daniel Markewitz ${ }^{1}$ has objected to the use of the term 'soil' for material recently studied remotely by Mars Sojourner, because he thinks soil should be defined as a medium for life. But there is a very compelling reason why the US National Aeronautics and Space Administration and many scientists choose to talk of soil on the surface of the Moon and Mars. They wish to be understood. Soil, like love and home, is difficult to define, but is, at the same time, a familiar and comforting concept $t^{2}$.

A geological as opposed to an engineering or agricultural definition of soil is material altered in place at the surface of a planetary body by physical, chemical or biological means ${ }^{3}$. This definition of soil introduces the idea of alteration in place as a distinction between soil and sediment. This important distinction is deliberately blurred by terms such as regolith and gradation, also used in planetary geology ${ }^{4}$, especially where only remotesensing data are available. But in martian images, sediment, which is bedded, can be distinguished from soil, which is clayey and salty ${ }^{3}$.

Gregory J. Retallack

Department of Geological Sciences,

University of Oregon,

Eugene,

Oregon 97402-1272, USA

e-mail:greg_retallack@ccmail.uoregon.edu

1. Markewitz, D. Nature 389, 435 (1997).

2. Hole, F. Geoderma 25, 75-112 (1981).

3. Retallack, G. J. Soils of the Past (Unwin-Hyman, London, 1990).

4. Greeley, R. Planetary Landscapes (Unwin-Hyman, London, 1985). 